\title{
Supplementary Information for:
}

\section{And yet it moves: $\mathrm{LiNiO}_{2}$, a dynamic Jahn-Teller system}

Sabrina Sicolo, ${ }^{* \dagger}{ }^{\dagger}$ Markus Mock, ${ }^{\dagger}$ Matteo Bianchini, ${ }^{\dagger}$ and Karsten Albe ${ }^{\ddagger}$

†BASF SE, Carl-Bosch-Strasse 38, 67056 Ludwigshafen am Rhein, Germany

$\ddagger$ Technische Universitaet Darmstadt, Institut fuer Materialwissenschaft, Fachgebiet

Materialmodellierung, Otto-Berndt-Str. 3, 64206 Darmstadt, Germany

E-mail: sabrina.sicolo@basf.com 


\section{Vibrational properties}
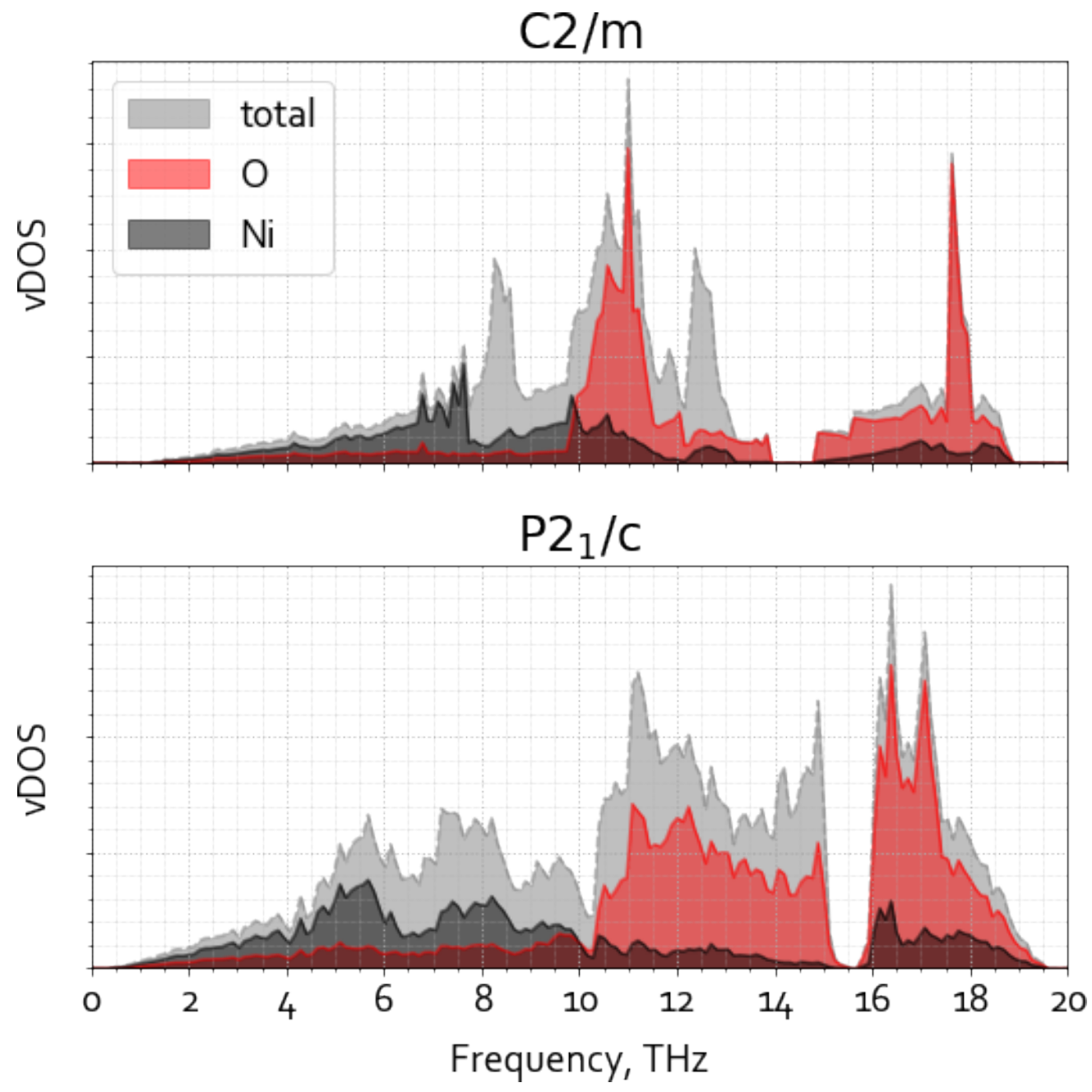

Figure S1: Vibrational densities of states for $\mathrm{P} 2_{1} / \mathrm{c}$ and $\mathrm{C} 2 / \mathrm{m}$. 
Table S1: Г-point eigenvalues, total and JT-specific zero-point vibrational energies for the unit cells of $\mathrm{P} 2{ }_{1} / \mathrm{c}$ and $\mathrm{C} 2 / \mathrm{m}$. JT-active modes are marked with a superscript.

\begin{tabular}{|c|c|c|}
\hline & $\mathrm{P} 2_{1} / \mathrm{c}$ & $\mathrm{C} 2 / \mathrm{m}$ \\
\hline$\omega_{1}, \mathrm{THz}$ & 18.06 & 18.14 \\
\hline$\omega_{2}, \mathrm{THz}$ & 17.82 & 18.01 \\
\hline$\omega_{3}, \mathrm{THz}$ & 17.47 & 16.59 \\
\hline$\omega_{4}, \mathrm{THz}$ & 17.45 & 13.07 \\
\hline$\omega_{5}, \mathrm{THz}$ & 16.47 & $11.34^{a}$ \\
\hline$\omega_{6}, \mathrm{THz}$ & 16.30 & $10.07^{a}$ \\
\hline$\omega_{7}, \mathrm{THz}$ & 14.12 & 9.97 \\
\hline$\omega_{8}, \mathrm{THz}$ & 14.08 & 7.84 \\
\hline$\omega_{9}, \mathrm{THz}$ & 13.01 & 7.74 \\
\hline$\omega_{10}, \mathrm{THz}$ & 12.69 & \\
\hline$\omega_{11}, \mathrm{THz}$ & $12.58^{a}$ & \\
\hline$\omega_{12}, \mathrm{THz}$ & $11.76^{a}$ & \\
\hline$\omega_{13}, \mathrm{THz}$ & $11.54^{a}$ & \\
\hline$\omega_{14}, \mathrm{THz}$ & $10.89^{a}$ & \\
\hline$\omega_{15}, \mathrm{THz}$ & 10.73 & \\
\hline$\omega_{16}, \mathrm{THz}$ & 10.55 & \\
\hline$\omega_{17}, \mathrm{THz}$ & 9.34 & \\
\hline$\omega_{18}, \mathrm{THz}$ & 7.44 & \\
\hline$\omega_{19}, \mathrm{THz}$ & 6.23 & \\
\hline$\omega_{20}, \mathrm{THz}$ & 5.66 & \\
\hline$\omega_{21}, \mathrm{THz}$ & 5.59 & \\
\hline $\mathrm{ZPE}, \mathrm{meV} / \mathrm{LiNiO}_{2}$ & 292 & 285 \\
\hline ZPE ${ }^{\mathrm{JT}}, \mathrm{meV} / \mathrm{LiNiO}_{2}$ & 48 & 44 \\
\hline
\end{tabular}

a JT-active modes. 


\section{Dynamics and cooperativity}

Table S2: Lengths of the six $\mathrm{Ni}-\mathrm{O}$ bonds of a $\mathrm{NiO}_{6}$ octahedron averaged over time and all $\mathrm{NiO}_{6}$ octahedra as a function of temperature.

\begin{tabular}{ccccccc}
\hline $\mathrm{T}, \mathrm{K}$ & $\overline{d_{1}}, \AA$ & $\overline{d_{2}}, \AA$ & $\overline{d_{3}}, \AA$ & $\overline{d_{4}}, \AA$ & $\overline{d_{5}}, \AA$ & $\overline{d_{6}}, \AA$ \\
\hline 300 & 1.943 & 1.942 & 1.944 & 1.943 & 1.942 & 1.942 \\
250 & 1.942 & 1.943 & 1.941 & 1.942 & 1.941 & 1.943 \\
200 & 1.941 & 1.942 & 1.942 & 1.941 & 1.942 & 1.941 \\
125 & 1.941 & 1.941 & 1.940 & 1.941 & 1.940 & 1.940 \\
50 & 1.939 & 1.943 & 1.937 & 1.941 & 1.938 & 1.941 \\
\hline
\end{tabular}



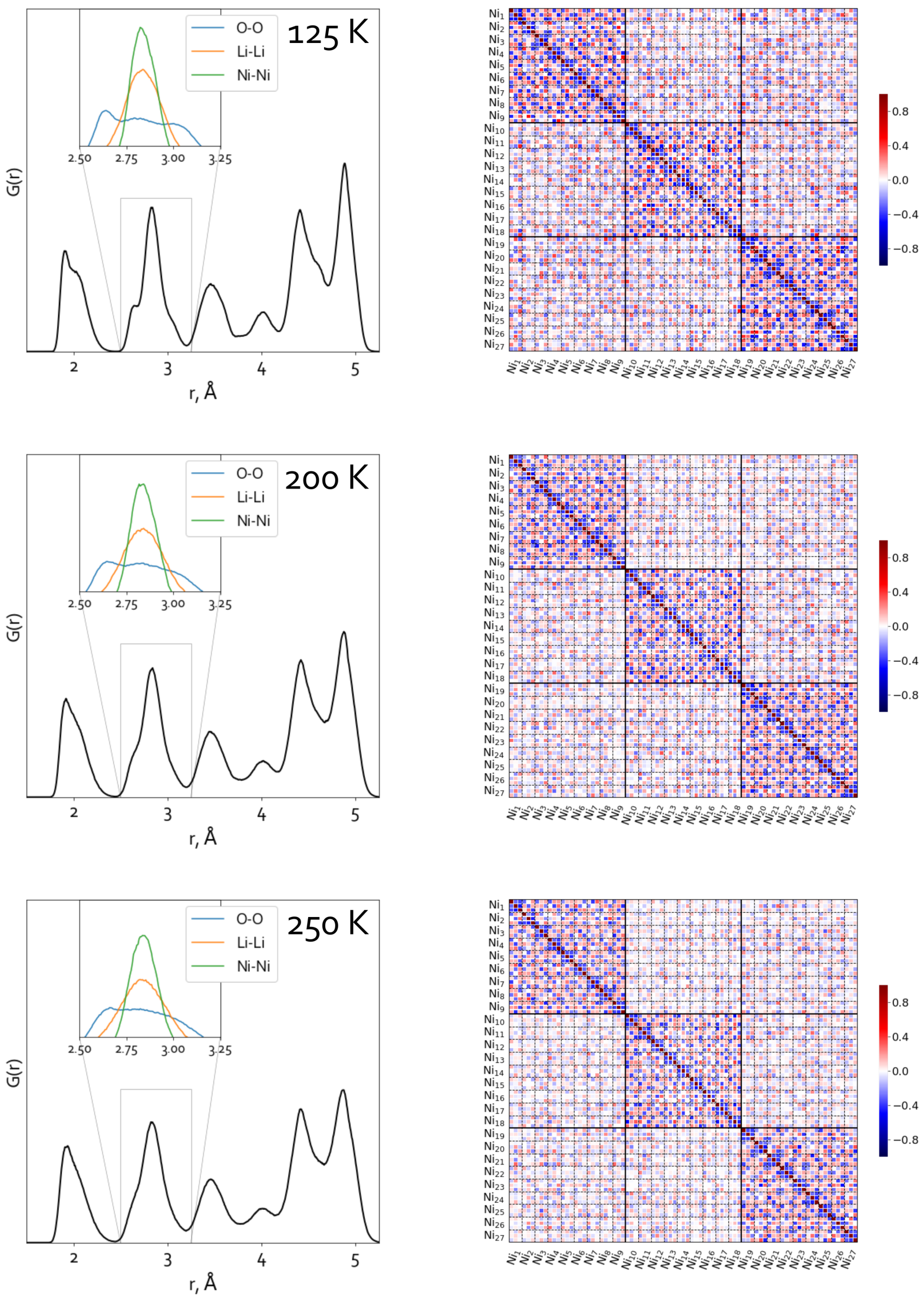

Figure S2: Correspondence between pair distribution functions and correlation matrices as a function of temperature. 


\section{Reorientation rates}

To dampen thermal oscillations of $\mathrm{Ni}-\mathrm{O}$ bond lengths and avoid overcounting flips, we applied a rolling average with a window of $100 \mathrm{fs}$ (Figure S3). A given bond was considered elongated if the difference to the second longest was at least $0.1 \AA$. These settings are the best match to our manual counting of flips based on chemical intuition and rather err on the conservative side of counting.

\section{raw data}
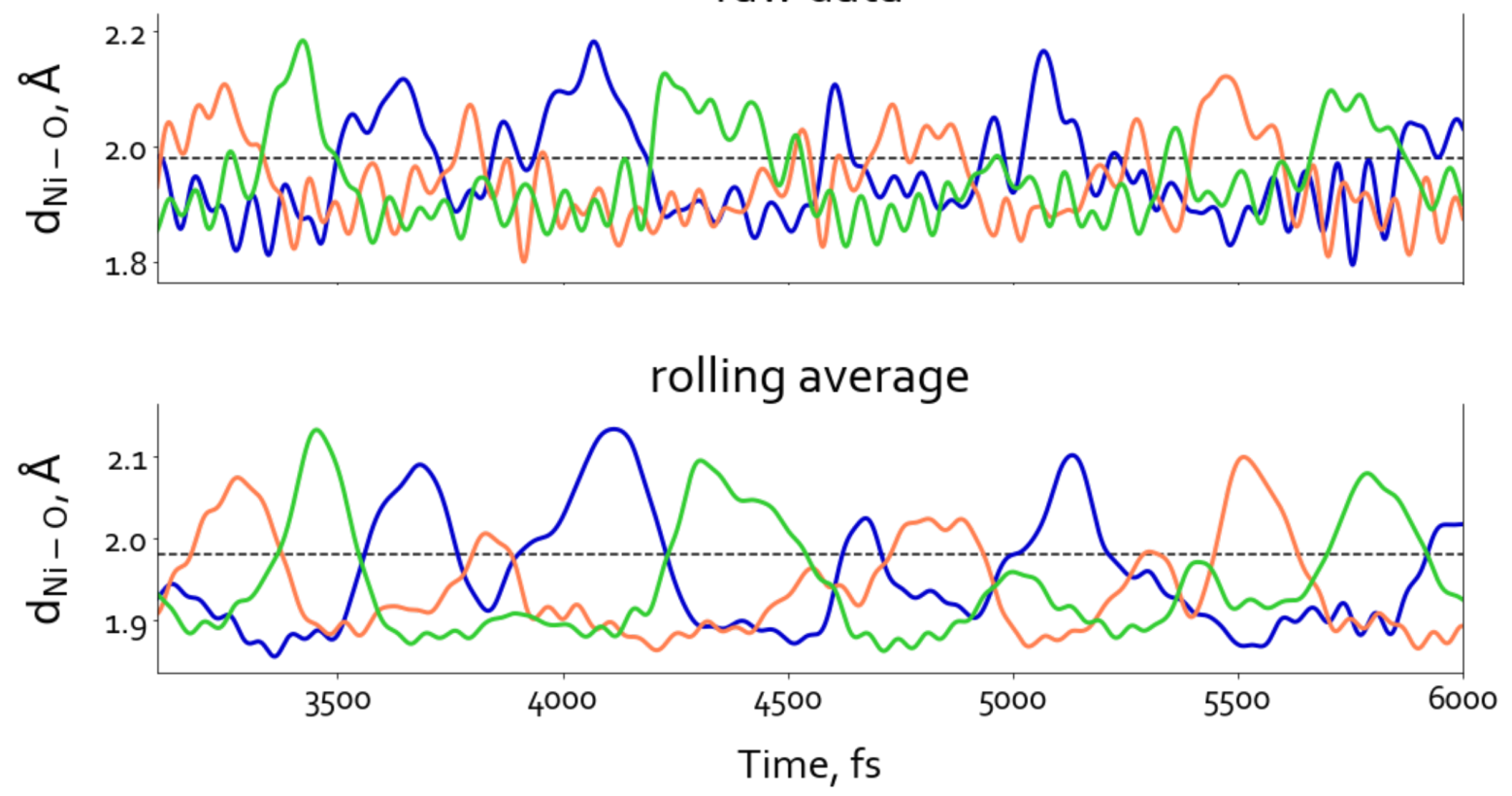

Figure S3: Comparison between raw $\mathrm{Ni}-\mathrm{O}$ bond lengths and their rolling averages as a function of time. 
We fitted several Arrhenius lines to our flip rates as a function of the reciprocal temperature on a semi-logarithmic scale (Figure S4). The fits differ in whether the lowest temperatures are considered. The grey region marks the area between the steepest and flattest lines and serves as an error bar for the fit.

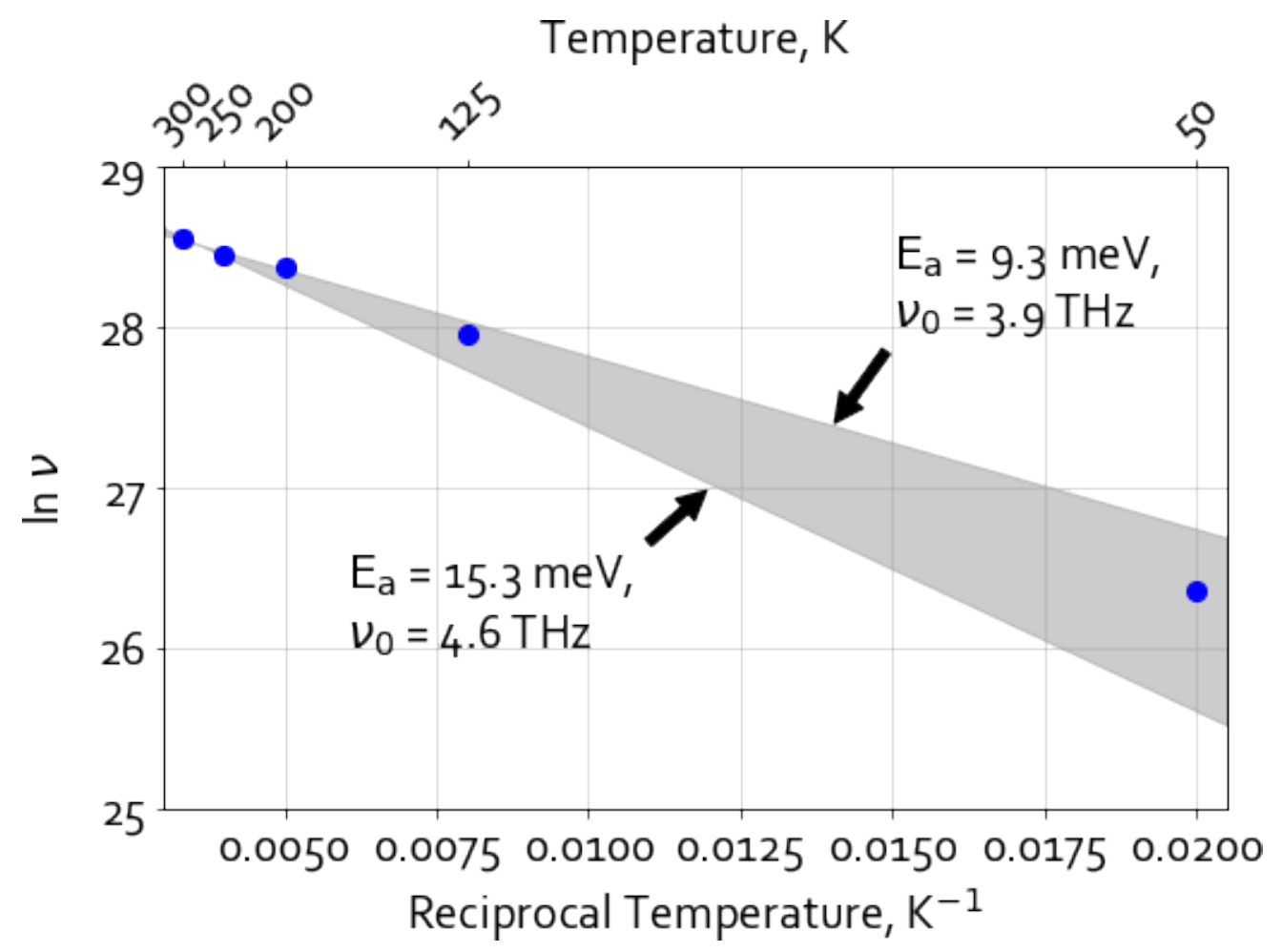

Figure S4: Computed reorientation rates as a function of temperature. Marked in grey is the area that comprises several Arrhenius fits to possible subsets of temperatures. 


\section{Sensitivity studies}

\section{Electronic structure}

Table S3: Relative stabilities in $\mathrm{meV} / \mathrm{f}$.u. of the collinear JT-distorted $(\mathrm{C} 2 / \mathrm{m})$ and disproportionated $(\mathrm{P} 2 / \mathrm{c})$ with respect to the ground state $\mathrm{P} 22_{1} / \mathrm{c}$ obtained with SCAN+rVV10 and $\mathrm{SCAN}+\mathrm{rVV} 10+\mathrm{U}(\mathrm{U}=2.5 \mathrm{eV})$.

\begin{tabular}{cccc}
\hline & $\mathrm{P} 2{ }_{1} / \mathrm{c}$ & $\mathrm{C} 2 / \mathrm{m}$ & $\mathrm{P} 2 / \mathrm{c}$ \\
\hline SCAN+rVV10 & 0 & 13 & 31 \\
SCAN+rVV10+U & 0 & 4 & 20 \\
\hline
\end{tabular}

Table S4: Comparison between the parameters of the potential energy landscape obtained with SCAN+rVV10 and SCAN+rVV10+U $(U=2.5 \mathrm{eV})$.

\begin{tabular}{cccc}
\hline & $E_{J T}, \mathrm{meV} /$ f.u. & $E_{J T^{\prime}}{ }^{\prime}, \mathrm{meV} /$ f.u. & ZPVE $^{\text {JT }}, \mathrm{meV} /$ f.u. \\
\hline SCAN+rVV10 & 70 & 22 & 48 \\
SCAN+rVV10+U & 108 & 63 & 45 \\
\hline
\end{tabular}

(a)

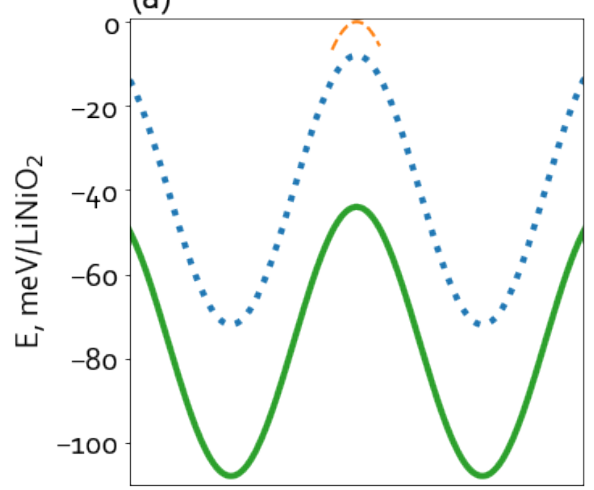

(b)

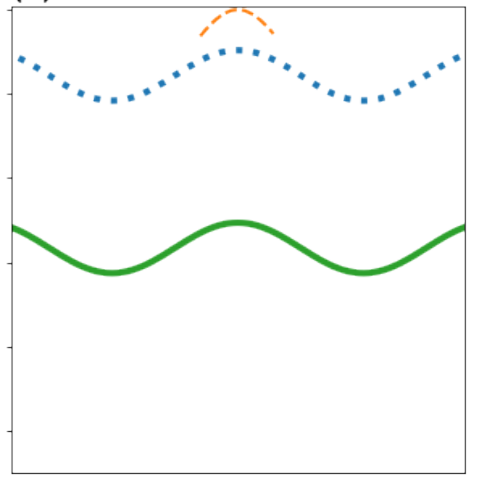

(c)

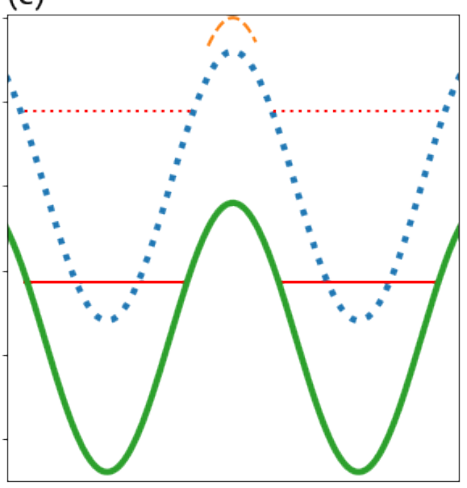

Figure S5: Sections of the potential energy surface calculated with SCAN+rVV10 (dotted blue line) and SCAN+rVV10+U $(U=2.5 \mathrm{eV}$, solid green line). The $y$ axis has been rescaled to show the high-symmetry point (dashed orange cusp) at 0. 


\section{Supercell size}

We performed the same molecular dynamics study on a $4 \times 4 \times 1$ supercell of the conventional rhombohedral cell. Calculated reorientation rates are in very good agreement with the ones in the main text (Figure S6 and Table S5). The correlation analysis at $300 \mathrm{~K}$ also returned a perfectly consistent result, showing partial intra-layer correlation that quickly decays between different layers (Figure S7).

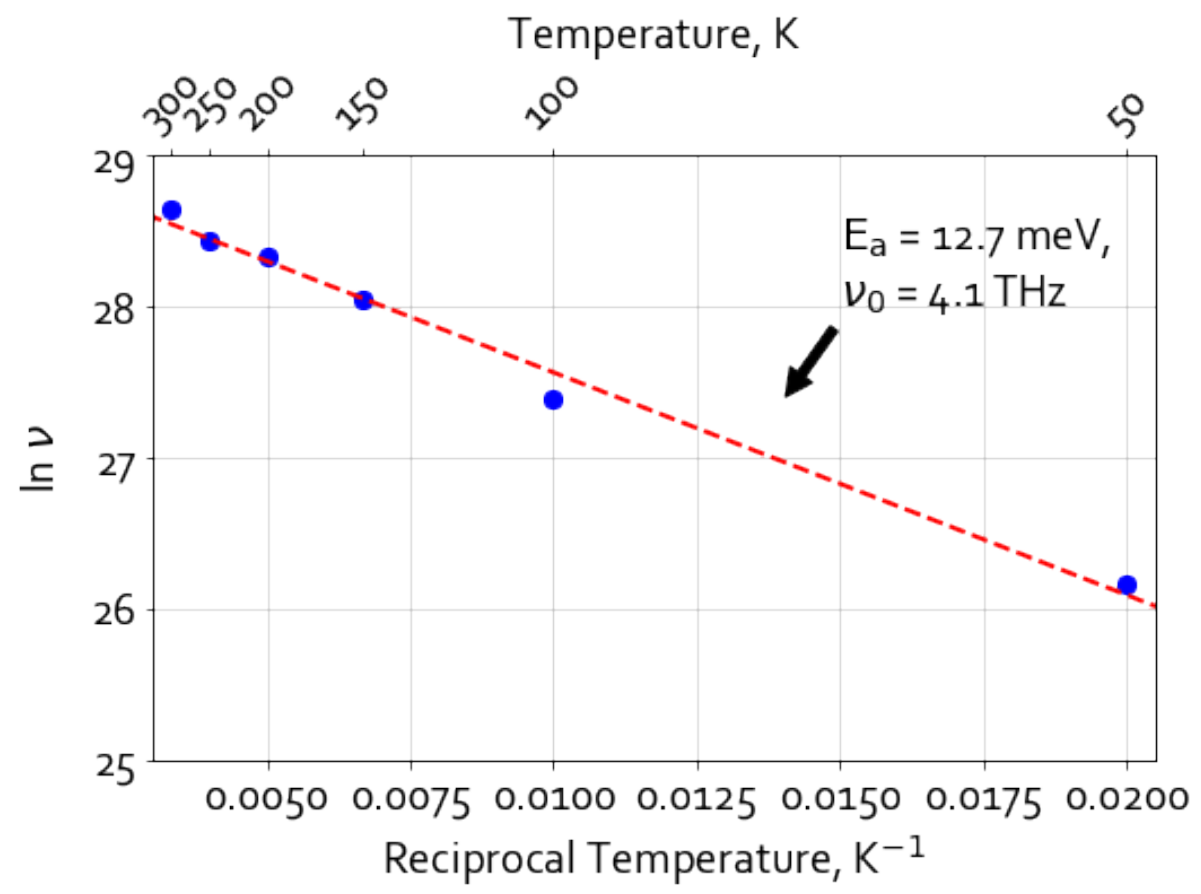

Figure S6: Rates for the reorientation of JT distortions in the $4 \times 4 \times 1$ supercell and corresponding Arrhenius fit. 


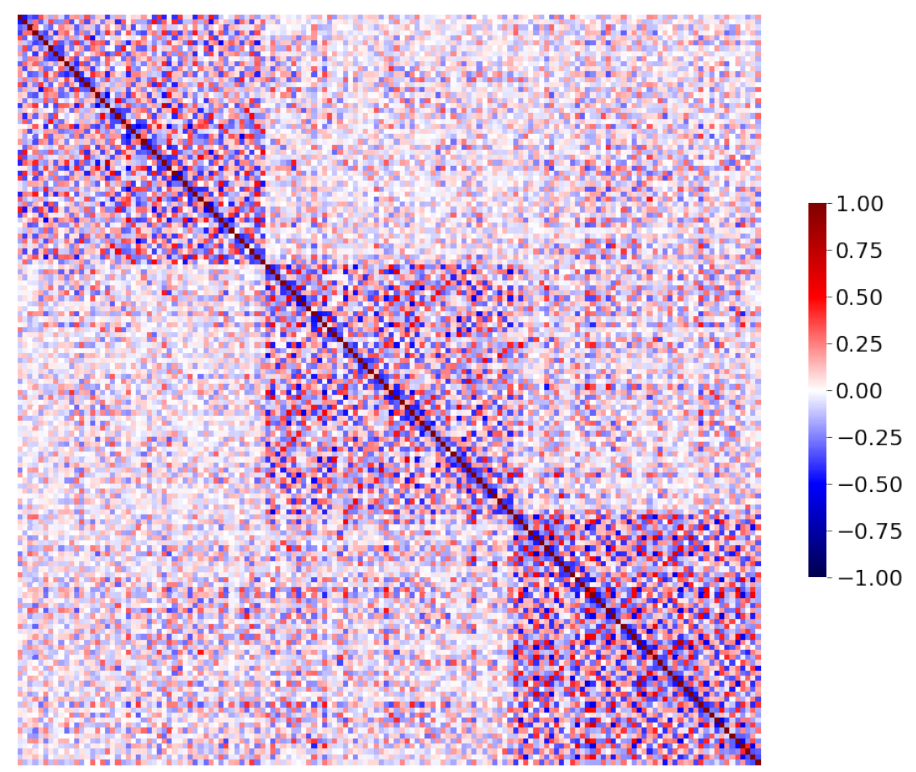

Figure S7: Correlation matrix for the $4 \times 4 \times 1$ supercell at $300 \mathrm{~K}$.

\section{Supercell symmetry}

We performed molecular dynamics also on a $2 \times 4 \times 2 \mathrm{P} 2{ }_{1} \mathrm{C}$ supercell to see how the ground state reacts to thermal motion. Also in this case, the JT directions reorient, but the fixed monoclinic shear freezes one of the three directions in its initial orientation. In Figure S8 we show the $\mathrm{P} 2{ }_{1} \mathrm{C}$ counterpart to Figure 3 in the main text. A rolling average has been applied to aid the visualization.

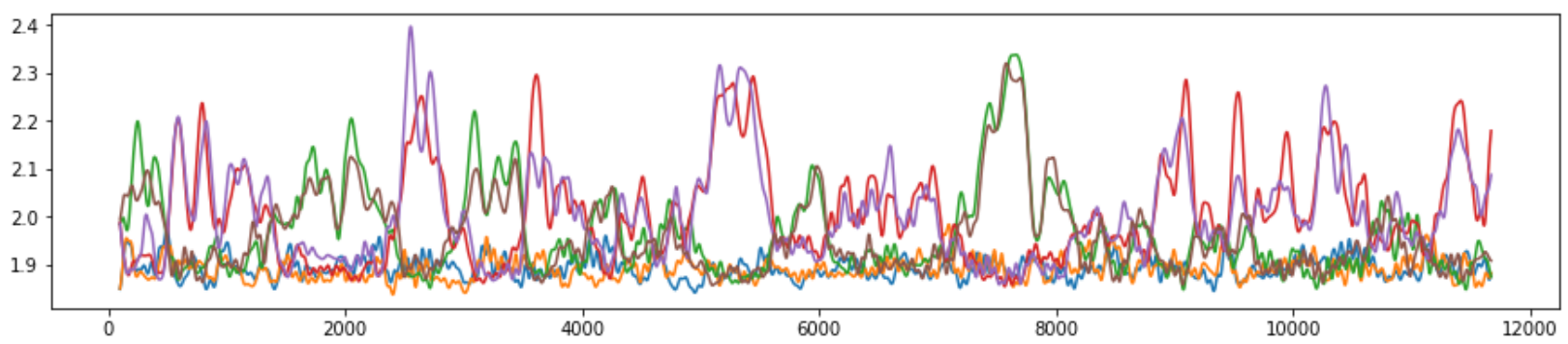

Figure S8: Rolling average of $\mathrm{Ni}-\mathrm{O}$ bond lengths for an arbitrary $\mathrm{NiO}_{6}$ octahedron in a $2 \times 4 \times 2$ $\mathrm{P} 2{ }_{1}$ c supercell. 


\section{Initial bonding configuration}

We initialized a rhombohedral $3 \times 3 \times 1$ supercell with JT-distorted bonds. The bonds were collinear within each layer, but the three layers were oriented along the three possible JT directions: this way, there was no tendency toward monoclinic shearing and the stress was minimized. Once again, the computed reorientation rates (at 250 and $300 \mathrm{~K}$ ) are consistent with the ones we presented in the main text (Table S5).

Table S5: Rates for the reorientation of JT distortions in a $4 \times 4 \times 1$ supercell and $3 \times 3 \times 1$ supercell with a JT-distorted starting configuration compared to the rates presented in the main text.

\begin{tabular}{llll}
\hline $\mathrm{T}, \mathrm{K}$ & $\nu_{3 \times 3 \times 1}, \mathrm{~Hz}$ & $\nu_{4 \times 4 \times 1}, \mathrm{~Hz}$ & $\nu_{3 \times 3 \times 1} \mathrm{JT}, \mathrm{Hz}$ \\
\hline 300 & $2.54 \cdot 10^{12}$ & $2.42 \cdot 10^{12}$ & $2.78 \cdot 10^{12}$ \\
250 & $2.26 \cdot 10^{12}$ & $1.99 \cdot 10^{12}$ & $2.24 \cdot 10^{12}$ \\
\hline
\end{tabular}

